\title{
Cost-Effectiveness Analysis of d-Nav for People with Diabetes at High Risk of Neuropathic Foot Ulcers
}

William Green · Matthew Taylor

Received: June 9, 2016 / Published online: July 11, 2016

(C) The Author(s) 2016. This article is published with open access at Springerlink.com

\section{ABSTRACT}

Introduction: The objective of this study was to assess the cost-effectiveness of the d-Nav Insulin Guidance Service (Hygieia Inc.), a system designed to improve glycemic control via the use of insulin titration, in people with diabetes at risk of developing neuropathic foot ulcers.

Methods: A Markov model containing four health states (no ulcer, uninfected ulcer, infected ulcer, and amputation) was developed to compare d-Nav with current National Health Service standard care. Patient movement between the health states was governed by event rates taken from the wider literature. Both the healing rate for uninfected ulcers and the rate of recurrence for uninfected ulcers were directly influenced by the patient's glycated hemoglobin (HbA1c). Separate mean HbA1c values were assigned to treatment and control patients and taken from a single-arm study that

Enhanced content To view enhanced content for this article go to http://www.medengine.com/Redeem/ C0E4F06075711211.

W. Green $(\bowtie) \cdot$ M. Taylor

University of York, York Health Economics

Consortium, York, UK

e-mail: william.green@york.ac.uk examined the effect of d-Nav on the outcomes of 122 patients, with $\mathrm{HbA1c}$ for control patients based on values recorded in the 12-month period prior to the study and $\mathrm{HbA} 1 \mathrm{c}$ for $\mathrm{d}-\mathrm{Nav}$ based on values recorded during the trial. Weekly cycles were applied, and patient resource use and quality-adjusted life years (QALYs) were estimated over a 3-year time horizon. Univariate sensitivity analysis was undertaken.

Results: In the base case, d-Nav was cost-saving and produced more QALYs than standard care, with a total net monetary benefit value of $£ 1459$ per patient. Univariate analysis indicated that the model results are relatively robust to variations in underlying parameters, with patient HbA1c having the most significant impact on outcomes.

Conclusion: Interventions that aim to improve glycemic control, such as d-Nav, appear to be a cost-effective use of healthcare resources when targeted at those with poor glycemic control at high risk of developing foot ulcers.

Funding: Hygieia Inc.

Keywords: Cost-effectiveness analysis;

Diabetes; d-Nav; Foot ulcer; Glycemic control 


\section{INTRODUCTION}

The major consequence of poor glycemic control in people with diabetes is chronic hyperglycemia [1], which can lead to a large number of microvascular and macrovascular issues. One common microvascular condition is foot ulceration, which results from diabetic neuropathy and vascular complications. The risk of developing neuropathy is directly proportional to both the duration and magnitude of hyperglycemia [2], and therefore, maintaining glycemic control is vital to reduce the number of foot ulcers. There are mixed data on the prevalence of foot ulcers, but estimations as high as $25 \%$ of patients with diabetes being affected over their lifetime, have been made [3]. The management of the condition is complex as a large proportion will fail to heal, and there is an increased risk of serious infection and extremity amputation [4]. Therefore, the condition is a significant financial burden on the National Health Service (NHS), through outpatient costs, increased bed occupancy and prolonged hospital stays [5]. The size of this burden is highlighted by a report by Hex and colleagues [6], who estimated the cost of foot ulcers and amputations to the NHS to be $£ 985 \mathrm{~m}$ in 2010/2011, the most expensive category of diabetes complications.

There is a well-established link between uncontrolled or high glycated hemoglobin (HbA1c) levels and clinical complications [7]. As such, it is important to keep HbA1c levels stable to reduce the risk of these complications, with guidance in the UK stating that HbA1c should be kept at a level of $6.5 \%(48 \mathrm{mmol} / \mathrm{mol})$ or below in people with type 1 diabetes, and between $6.5 \%$ and $7 \%(48-53 \mathrm{mmol} / \mathrm{mol})$ in people with type 2 diabetes $[8,9]$. However, in practice, patients often fail to achieve suitable HbA1c levels, as shown by a survey in Sweden that found only less than one-third of insulin-treated patients with type 2 diabetes to havereached the recommended HbA1c goal of $<7 \%$ (53 mmol/mol), over 6 years [10]. Overall, there is mixed evidence regarding the mean HbA1c level among the diabetes population. However, two large-scale meta-analyses of diabetes-related interventions found that, among participants, baseline HbA1c was $8.05 \%$ and $8.13 \%$, indicating that mean levels are likely to be at least $8 \%(64 \mathrm{mmol} / \mathrm{mol})[11,12]$.

Insulin therapy is seen as an important treatment option for both type 1 and type 2 diabetes $[13,14]$. Administration is commonly achieved via manual insulin injection or insulin pumps, supported by self-monitoring of blood glucose. However, this method has been used for many years, and the problem of poor glycemic control remains. This issue is highlighted by a paper by Simmons and colleagues [15], which found that $63 \%$ of pump users (916 participants) and $70 \%$ of injection users (978 participants) had a HbA1c of $8.5 \%(69 \mathrm{mmol} / \mathrm{mol})$ or greater. More effective glycemic control can be achieved using an insulin titration protocol. These protocols allow for more sensitive adjustments in insulin doses, leading to both near-normal glucose readings and the avoidance of hypoglycemic events [16]. However, insulin titration is complex, so achieving these goals is a difficult task. The difficulty largely arises as adjustments to insulin dosage must currently be undertaken with the assistance of a physician. However, visits usually occur every 3-6 months, making the system of titration unresponsive to short-term changes in blood glucose. Bastyr and colleagues [17] analyzed the impact of intensive insulin therapy, accompanied by monitoring 
using an electronic diary. The study found that HbA1c fell over the 26-week investigation period, in line with similar trials. The study also found that a mean number of 98.5 insulin adjustments per patient year were required for patients with type 1 diabetes, and 112.5 required for patients with type 2 diabetes. These adjustments were largely clinician-driven, indicating the high levels of resource use required [17].

To overcome this problem, the Diabetes Insulin Guidance Service (DIGS ${ }^{\mathrm{TM}}$, Hygieia Inc.) has been developed. This service aims to optimize patients' insulin dosage regimens. This is achieved through the use of algorithms that identify patterns in time-lagged glucose readings and adjust insulin dosage accordingly. DIGS is operational via a handheld device, known as $\mathrm{d}-N a v^{\circledR}$ (Hygieia Inc.), which also includes an in-built glucose sensor (see Fig. 1). Because this process is automated, it facilitates the unsupervised alteration of insulin dosage [16]. Therefore, suitable patients can be prescribed a d-Nav device to use on a daily basis to control their

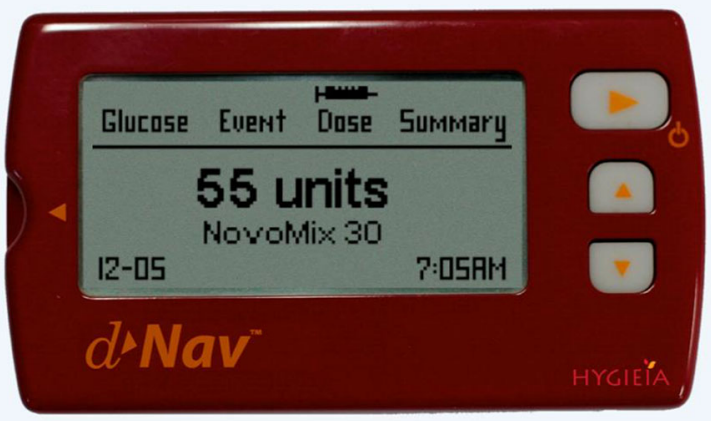

Fig. 1 Example of d-Nav device. The Diabetes Insulin Guidance System has been designed to optimize patients' insulin dosage regimens through the use of algorithms that identify patterns in time-lagged glucose readings and adjust insulin dosage accordingly. This is achieved using a handheld device (d-Nav), which is shown here, and is supported by a nurse-based service to ensure good use insulin regimen. As d-Nav aims for more sensitive adjustments in insulin, this should lead to improved glycemic stability, particularly in patients with high HbA1c levels. The device is supported by a nurse-based service to ensure that d-Nav is being used correctly, with communication possible both in person and by phone. Patient readings are also saved on an online database that can be reviewed by the patient's regular physician to ensure that the service is linked to the wider healthcare system.

In this paper, the cost-effectiveness of the d-Nav service for patients with poor glycemic control is evaluated from the perspective of the UK NHS. The d-Nav service is compared with current standard care in which patients do not have access to the d-Nav service and, therefore, any adjustments to their insulin regimen must be made via usual follow-up appointments with their regular physician.

\section{METHODS}

\section{Population}

The target population for this analysis was patients with diabetes currently treated by the UK NHS who are at high risk of foot ulcers, defined as those who have a HbA1c of $9 \%$ or greater $(74.9 \mathrm{mmol} / \mathrm{mol})$. HbA1c is the main driver as it has been established that it directly influences both the healing rate for foot ulcers and the rate of wound recurrence $[18,19]$. Two patient groups were included: those receiving d-Nav to control HbA1c (i.e., the treatment group), and those receiving current standard NHS diabetes care (i.e., the control group). For this analysis, NHS standard care is defined as the administration of insulin via injection or pumps and accompanied by self-monitoring of blood glucose. In practice, patients with 
diabetes would receive a greater range of therapies, but this distinct population has been chosen here, as these are the patients most applicable to the d-Nav service. The underlying HbA1c for patients in both groups was determined by a single-arm observational study, which examined the effectiveness of d-Nav to improve glycemic control [20]. This was a single-center evaluation in which 122 patients were trained to use d-Nav as part of their usual treatment protocol and, then, followed for 12 months, with the primary outcome measures being change in HbA1c. Ninety-four patients completed the full 12-month service evaluation. HbA1c was also investigated in all patients in the 12 months prior to study initiation and remained stable throughout. At study initiation, the mean HbA1c was $9.2 \pm 1.4 \%$. During the study, mean HbA1c decreased to $7.8 \pm 1.2 \%$ at 3-5 months and, further, to $7.5 \pm 1.2 \%$ at 6-12 months.
It was assumed that throughout the period of the analysis, d-Nav patients had a stable HbA1c that was equivalent to patients at the end of the study period (i.e., $7.5 \pm 1.2 \%$ ), while control patients had a stable HbA1c that was equivalent to patients at study baseline (i.e., $9.2 \pm 1.4 \%$ ). In reality, over the long term, d-Nav may reduce HbA1c further, as shown in Fig. 2. Three year data has recently been presented by Dr Harper and colleagues at the 2016 American Diabetes Association conference, which confirmed that HbA1c with d-Nav remained stable at $7-7.5 \%$ over three years [21]. This data was presented after this analysis was completed, and therefore, the data were not formally included. To more accurately model the glycemic level of patients, these mean and standard deviation values were used to estimate the distribution of patients across six HbA1c levels (<6\%, 6-7\%, 7-8\%, 8-9\%, 9-10\%, and $>10 \%$ ). A normal distribution was assumed. The number of patients in each level, for both the treatment and control groups, is shown in Fig. 3.

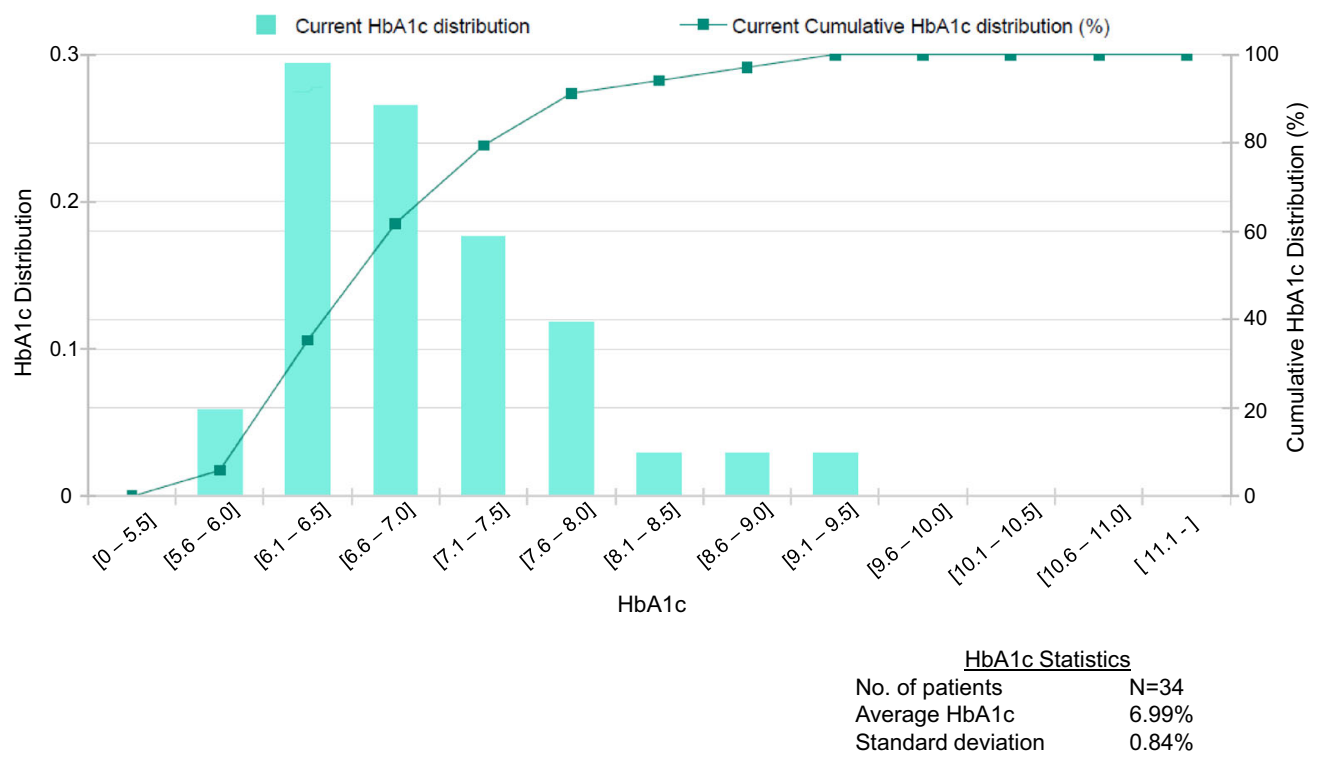

Fig. 2 Subscribers on d-Nav service for $27+$ months. All UK-based users of the d-Nav service have their HbAlc levels recorded as part of their treatment regimen. The results of 34 patients who have recorded data for at least
27 months are presented here (data provided by Hygieia Inc., the manufacturer of the device). It shows that over the long term, d-Nav users have a mean HbAlc of less than $7 \%$ 


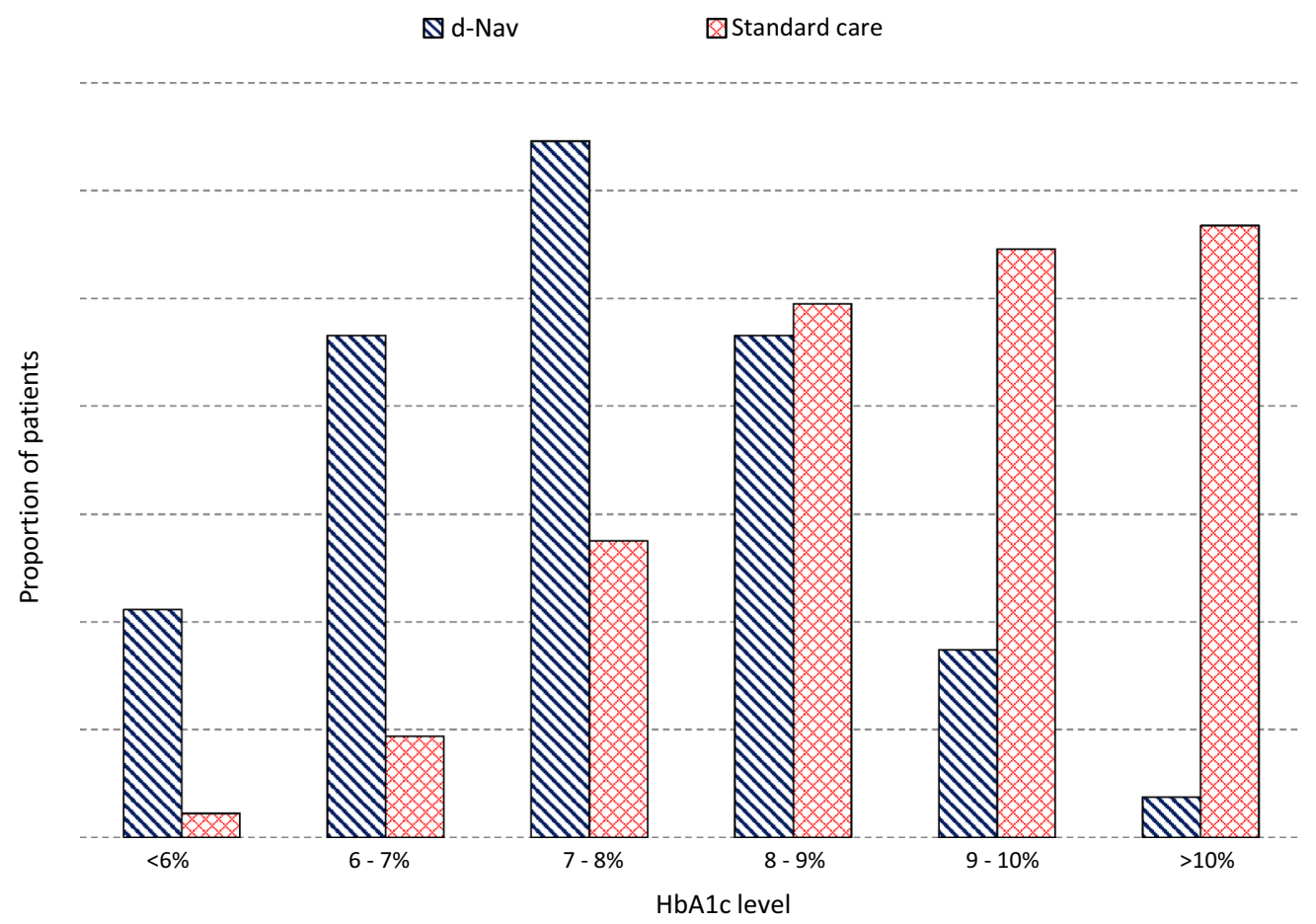

Fig. 3 Distribution of patients by HbAlc level. Within the analysis, patient HbAlc levels impact on the healing rate for diabetic foot ulcers, with higher HbAlc levels associated with lower rates of healing. The distribution of

Patients are removed from the d-Nav service if their HbA1c levels do not reduce after the first 3 months, as it is deemed that they are not benefiting. It was assumed that the proportion of patients with a HbA1c of $\geq 9 \%$, as determined by the underlying HbA1c distribution for d-Nav patients, would stop at month three. This equated to $10.56 \%$ of d-Nav patients, and, in the model, these patients incurred the cost of d-Nav for the first 3 months and then reverted to control group costs for the remaining time in the model.

\section{Model Structure}

The cost-effectiveness of d-Nav was determined using a Markov model (Fig. 4), which was constructed using Microsoft Excel (Microsoft Corporation, Redmond, WA, USA). Markov
d-Nav and standard care patients across six HbAlc categories is presented below. The distribution is based on mean and standard deviation HbAlc values recorded by Donnelly et al. [20]

models are commonly used in economic evaluations to model long-term patient outcomes and are particularly applicable for progressive chronic conditions such as diabetes. Within Markov models, patients are allocated to predefined health 'states', with each state associated with specific costs and health outcomes. The current model contained four health states: no ulcer, uninfected ulcer, infected ulcer and amputation. Markov models operate in cycles, which cover a discrete time period (e.g., 1 month, 1 year), with weekly cycles adopted here. Patients remain in a set health state for one cycle and can transition to a separate state at the end of each cycle. Transition probabilities are used to predict patient movement between health states; for example, if a patient is treated with a more efficacious intervention, they will have a lower 

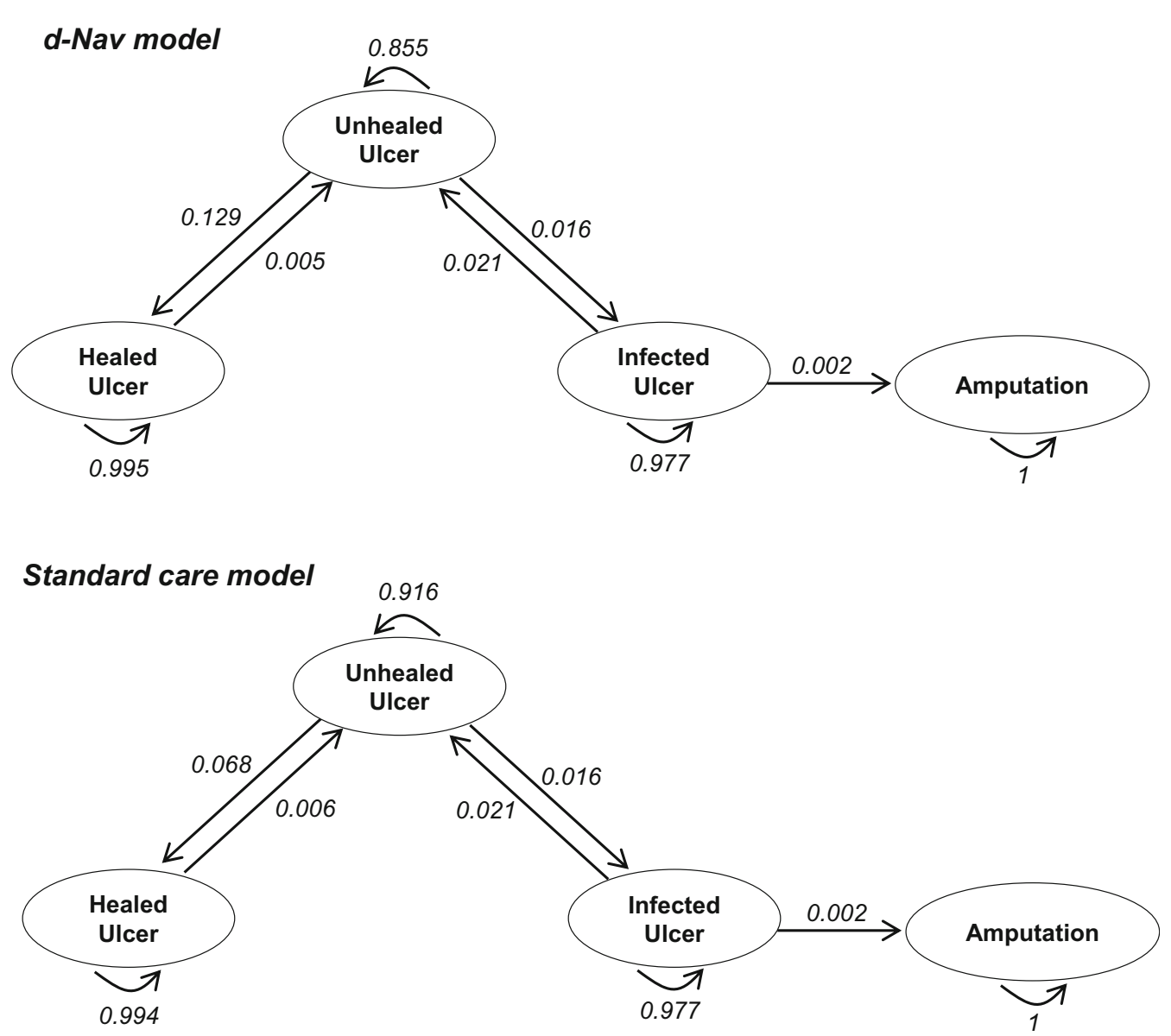

Fig. 4 Outline of Markov model structure. A Markov model was adopted to predict long-term patient outcomes with d-Nav and standard care. To capture the impact of diabetic foot ulcers on healthcare system costs and patient outcomes, four health states were included in the model: healed ulcer, unhealed ulcer, infected ulcer and

probability of transitioning from a better to a worse health state. As patients accumulate costs and health outcomes associated with the states, they reside in overtime, then remaining in a better health state leads to fewer costs and improved health outcomes.

In the current model, patients started in either the 'no ulcer' or 'uninfected ulcer' state. The distribution of patients between these two states was determined by the underlying prevalence of foot ulcers. It has been established that the prevalence of foot ulcers is $4.8 \%$ across the whole diabetes population, and this value amputation. The amputation state was absorbing such that once patients enter this state, they remained there for the full time horizon. Specific costs and health outcomes were allocated to each health state. The model structure and transition probabilities for each treatment option are shown

increases to $12.8 \%$ for patients aged 65 and over [22]. These figures cover all patients with diabetes, including those who have good glycemic control. It is, therefore, reasonable to assume that prevalence will be greater in the high-risk population considered in this analysis. However, an exact value for this parameter could not be identified. As such, a conservative assumption of $15 \%$ was adopted, and variations to this parameter were assessed during sensitivity analysis. Patients could move between the different states as outlined in Fig. 4. Once patients entered the amputation state, it was 
assumed that they would remain in that state for the remainder of the time in the model. In total, the model covered a 3-year time period, and this length of time was chosen due to the transient nature of foot ulcers. Within this 3-year period, mortality would be negligible with no discernible difference between treatment groups expected. Therefore, mortality was not considered.

\section{Transition Probabilities}

Movement between the health states was governed by transition probabilities, which were taken from the wider literature (Table 1). Due to the short timeframe used, these probabilities were not time-dependent. HbA1c directly influences both the healing rate and the rate of wound recurrence for foot ulcers. Christman et al. [18] stratified 183 individuals with diabetes into three HbA1c groups $(<7 \%$, $7-8 \%$, and $>8 \%$ ) and investigated the change in wound area $\left(\mathrm{cm}^{2}\right.$ per day). The investigators found that, for each $1 \%$ point increase in HbA1c, daily change in wound area decreased by $0.022 \mathrm{~cm}^{2}(P=0.027)$. They concluded that glycemic control is directly correlated with the healing rate for foot ulcers. In the model, daily change in wound area was converted to daily healing rate by dividing the change in area by the baseline area size for each of the three HbA1c categories. These healing rates were then converted to weekly transition probabilities, using the equation outlined in Fig. 5 and applied to each of the six HbA1c levels discussed previously. The HbA1c categories used in the model did not perfectly match those assessed by Christman et al. [18]. Therefore, the healing rate for patients with HbA1c $<7 \%$ was applied to both $<6 \%$ and $6-7 \%$ patients, while the healing rate for patients with HbA1c $>8 \%$ was applied to $8-9 \%, 9-10 \%$, and $>10 \%$ patients.
Table 1 A Summary of model effectiveness data

\begin{tabular}{lll}
\hline Parameter & Value & $\begin{array}{l}\text { Weekly Source } \\
\text { transition } \\
\text { probability }\end{array}$ \\
\hline
\end{tabular}

Daily healing rate

$\begin{array}{lll}\text { HbAlc }<6 \% & 3.24 \% & 0.206 \\ \text { HbA1c 6-7\% } & 3.24 \% & 0.206 \\ \text { HbA1c 7-8\% } & 3.24 \% & 0.206 \\ \text { HbA1c 8-9\% } & 2.06 \% & 0.135 \\ \text { HbA1c 9-10\% } & 0.68 \% & 0.047 \\ \text { HbA1c >10\% } & 0.68 \% & 0.047\end{array}$

Rate of wound recurrence

$\begin{array}{lll}\text { HbAlc }<7.5 \% & 33.3 \% & \text { N/A } \\ \text { HbAlc }>7.5 \% & 67.3 \% & \text { N/A }\end{array}$

Rate of wound recurrence

\begin{tabular}{llll} 
Treatment & $50.30 \%$ & 0.0045 & Calculated \\
Control & $62.99 \%$ & 0.0064 & Calculated \\
$\begin{array}{l}\text { Risk of wound } \\
\text { infection }\end{array}$ & $17.9 \%$ & 0.0163 & {$[28]$} \\
$\begin{array}{l}\text { Risk of amputation } \\
\text { Probability that }\end{array}$ & $8.7 \%$ & 0.017 & {$[29]$} \\
infection heals & 0.082 & 0.0212 & {$[26]$} \\
Utility & & & \\
No ulcer & 0.600 & N/A & {$[26]$} \\
Uninfected ulcer & 0.465 & N/A & {$[26]$} \\
Infected ulcer & 0.465 & N/A & {$[26]$} \\
Amputation & 0.450 & N/A & {$[26]$} \\
\hline
\end{tabular}

A number of input parameters were utilized in the model to predict long-term patient outcomes. These parameters are summarized below, along with the source of the information. Where applicable, rates were converted to probabilities to make the data applicable to the model structure adopted for the analysis. Both the original rate and subsequent transition probability are presented

Dubský et al. [19] followed 73 patients with diabetes over a 3-year time period to examine the frequency of, and risk factors for, foot ulcer 


$$
p=1-e^{\operatorname{Ln}(1-r / t)}
$$

where: $\quad p$ is the transition probability $r$ is the event rate $t$ is the time period

Fig. 5 Equation for converting event rates to transition probabilities. Event rates, such as the rate of ulcer healing, must be converted to probabilities to be applicable to Markov models. Within the analysis, rates were converted to probabilities using the equation outlined below

recurrence, with HbA1c being significantly associated with the risk of ulceration recurrence. Using data from the paper by Dubský et al. [19], it was estimated that the 3 -year risk of recurrence was $33.3 \%$ for patients with $\mathrm{HbA} 1 \mathrm{c}<7.5 \%$ and $67.3 \%$ for patients with HbA1c $>7.5 \%$. These rates were applied to the patient distributions to generate predicted 3-year healing rates. To be suitable for the Markov model, these healing rates were also converted to transition probabilities (Fig. 5).

Rate of infection, healing rate for infected ulcers and risk of amputation were also required. These parameters were not linked to patient HbA1c due to a deficiency in relevant data from the wider literature. Therefore, transition probabilities were estimated from relevant values identified in a targeted literature search and applied equally across all HbA1c groups.

\section{Costs and Resource Use}

Where necessary, costs were inflated to 2013/14 prices using the hospital and community health services index [23]. The insulin regimen considered in this analysis is once-daily long-acting insulin and short-acting insulin with meals, as it is the regimen most commonly used by d-Nav patients. Daily insulin usage was based on unpublished data from the observational d-Nav study. Overall, insulin usage was significantly higher in d-Nav patients. A large number of oral anti-diabetic agents were also included within the model. With d-Nav, patients are not required to take any agents except for insulin and metformin and, also, do not require the use of a blood glucose monitoring device as glucose tests are conducted with d-Nav. Therefore, total usage for glucose test strips and all anti-diabetic agents, except metformin, remained at zero for the treatment group. Usage among the control group was determined using data from the Northern Irish South Eastern Health and Social Care Trust. The Trust provided data on anti-diabetic agent usage from a sample of 3665 patients with type 2 diabetes (see Table 2). It was assumed that $100 \%$ of control group patients used a blood glucose monitoring device, in line with the patient population deemed most applicable for d-Nav, and they self-tested, on average, three times per day at a mean cost of $£ 14.57$ for 50 test strips [24]. This was combined with an average cost of $£ 3.42$ for 100 lancets equating to a weekly cost of $£ 6.84$ [25]. The cost of the d-Nav service is altered depending on the total number of users within each population, as outlined in Table 3.

Patients also incurred costs depending on the health state within which they resided (Table 3). These health state costs were sourced from the literature [4] and applied to weekly cycles.

\section{Utility}

To estimate the impact of treatment on patient health-related quality of life, quality-adjusted life year (QALY) scores were calculated using utility values. Utility is a measurement of patient's preferences for different health 
Table 2 Anti-diabetic drug usage in standard care

\begin{tabular}{lcc}
\hline $\begin{array}{l}\text { Anti-diabetic } \\
\text { agent }\end{array}$ & $\begin{array}{l}\text { Number } \\
\text { of patients } \\
\text { on drug }\end{array}$ & $\begin{array}{l}\text { Proportion } \\
\text { on drug (\%) }\end{array}$ \\
\hline Metformin & 3336 & 91.02 \\
Sitagliptin & 629 & 17.16 \\
Saxagliptin & 93 & 2.54 \\
Linagliptin & 224 & 6.11 \\
Vildagliptin & 158 & 4.31 \\
Pioglitazone & 383 & 10.45 \\
Dapagliflozin & 146 & 3.98 \\
Liraglutide & 714 & 19.48 \\
Exenatide & 179 & 4.88 \\
Acarbose & 14 & 0.38 \\
Repaglinide & 7 & 0.19 \\
Nateglinide & 6 & 0.16 \\
Metformin/Pioglitazone & 19 & 0.52 \\
Metformin/Vildagliptin & 125 & 3.41 \\
Metformin/Sitagliptin & 63 & 1.72 \\
Metformin/Linagliptin & 5 & 0.14 \\
\hline Patents with diabes & 5 &
\end{tabular}

Patients with diabetes may be treated with an anti-diabetic agent. In clinical practice, as part of the d-Nav service, all patients are required to use metformin but no other anti-diabetic agent. The values presented in the table show the numbers and proportion of patients in the standard care receiving each of the oral anti-diabetic agents. These values were taken from a sample of drug usage for 3665 patients with type 2 diabetes with data provided by South Eastern Health and Social Care Trust. The proportion of patients receiving each drug was applied to standard care patients in the analysis

outcomes. It uses a cardinal scale in which zero represents death and one represents full health. Utility values can be combined with estimates of survival, to calculate QALY scores, which combine both morbidity and mortality into a single outcome measure. A QALY score of one is equivalent to 1 year of life in full health. QALYs enable a consistent measurement of health benefit across different treatment options. This is particularly applicable when a new treatment option is expected to generate extra health benefits compared to current standard care but at a greater cost. In the analysis, utility values were based on the four health states. Values were sourced from the literature and applied to each weekly cycle [26]. To generate the QALY values, cumulative utility scores were divided by the total number of weeks elapsed.

\section{Sensitivity Analysis}

To test uncertainty within the model, univariate sensitivity analysis was undertaken. Underlying parameter values were varied to examine the impact on model outputs, as measured by net monetary benefit. For the majority of parameters, the original value was altered by $\pm 50 \%$ to robustly examine the effects of changes. Utility scores for all health states, and mean HbA1c for both treatment and control patients, were varied within smaller ranges as $\pm 50 \%$ was deemed to generate unrealistic values. The extreme values (high and low) tested for each parameter are shown in Fig. 6. Two cases of scenario analyses were also undertaken. First, the impact of incorporating only those patients with foot ulcers was examined (i.e., all patients started the model in the uninfected state, and this equated to $15 \%$ of the overall cohort). Second, a scenario was created in which the distribution of control patients' HbA1c levels was fixed, so that all patients had a HbA1c of $\geq 9 \%$. This is the optimal population to be targeted with the intervention.

\section{Evaluation}

Costs and utility values used in the model were both discounted at a rate of $3.5 \%$, in line 
Table 3 Resource use and health state costs

\begin{tabular}{|c|c|c|}
\hline Parameter & Weekly cost & Source \\
\hline \multicolumn{3}{|l|}{ Health states } \\
\hline No ulcer & $£ 5.21$ & {$[4]$} \\
\hline Uninfected ulcer & $£ 463$ & {$[4]$} \\
\hline Infected ulcer & $£ 460$ & {$[4]$} \\
\hline Amputation & $£ 818$ & {$[4]$} \\
\hline \multicolumn{3}{|l|}{ d-Nav Service } \\
\hline Installation fee & $£ 102.17$ & Communication with service provider \\
\hline \multicolumn{3}{|l|}{ Service fee } \\
\hline $0-50$ users & $£ 44.96$ & Communication with service provider \\
\hline $51-100$ users & $£ 40.46$ & Communication with service provider \\
\hline $101-200$ users & $£ 36.67$ & Communication with service provider \\
\hline$>200$ users & $£ 33.72$ & Communication with service provider \\
\hline \multicolumn{3}{|l|}{ Anti-diabetic drugs } \\
\hline Metformin & $£ 0.25$ & {$[31]$} \\
\hline Sitagliptin & $£ 9.83$ & {$[31]$} \\
\hline Saxagliptin & $£ 8.80$ & {$[31]$} \\
\hline Linagliptin & $£ 8.75$ & {$[31]$} \\
\hline Vildagliptin & $£ 7.63$ & {$[31]$} \\
\hline Pioglitazone & $£ 3.88$ & {$[31]$} \\
\hline Dapagliflozin & $£ 10.39$ & {$[31]$} \\
\hline Liraglutide & $£ 15.36$ & {$[31]$} \\
\hline Exenatide & $£ 20.31$ & {$[31]$} \\
\hline Acarbose & $£ 2.19$ & {$[31]$} \\
\hline Repaglinide & $£ 3.53$ & {$[31]$} \\
\hline Nateglinide & $£ 6.69$ & {$[31]$} \\
\hline Metformin/Pioglitazone & $£ 10.65$ & {$[31]$} \\
\hline Metformin/Vildagliptin & $£ 9.64$ & {$[31]$} \\
\hline Metformin/Sitagliptin & $£ 10.26$ & {$[31]$} \\
\hline Metformin/Linagliptin & $£ 9.52$ & {$[31]$} \\
\hline \multicolumn{3}{|l|}{ Insulin regimens } \\
\hline Lantus ${ }^{\circledR}$ (long-acting) & $£ 30.68$ per 1000 units & {$[30]$} \\
\hline
\end{tabular}


Table 3 continued

\begin{tabular}{lll}
\hline Parameter & Weekly cost & Source \\
\hline Apidra $^{\circledR}$ (short-acting) & $£ 16.00$ per 1000 units & {$[30]$} \\
Humulin M3 ${ }^{\circledR}$ (biphasic) & $£ 15.68$ per 1000 units & {$[30]$} \\
Blood glucose monitoring & $£ 6.84$ & {$[24,25]$} \\
\hline
\end{tabular}

All costs that have been applied within the analysis are summarized in the table, with the original source also given. All costs are weekly and per patient

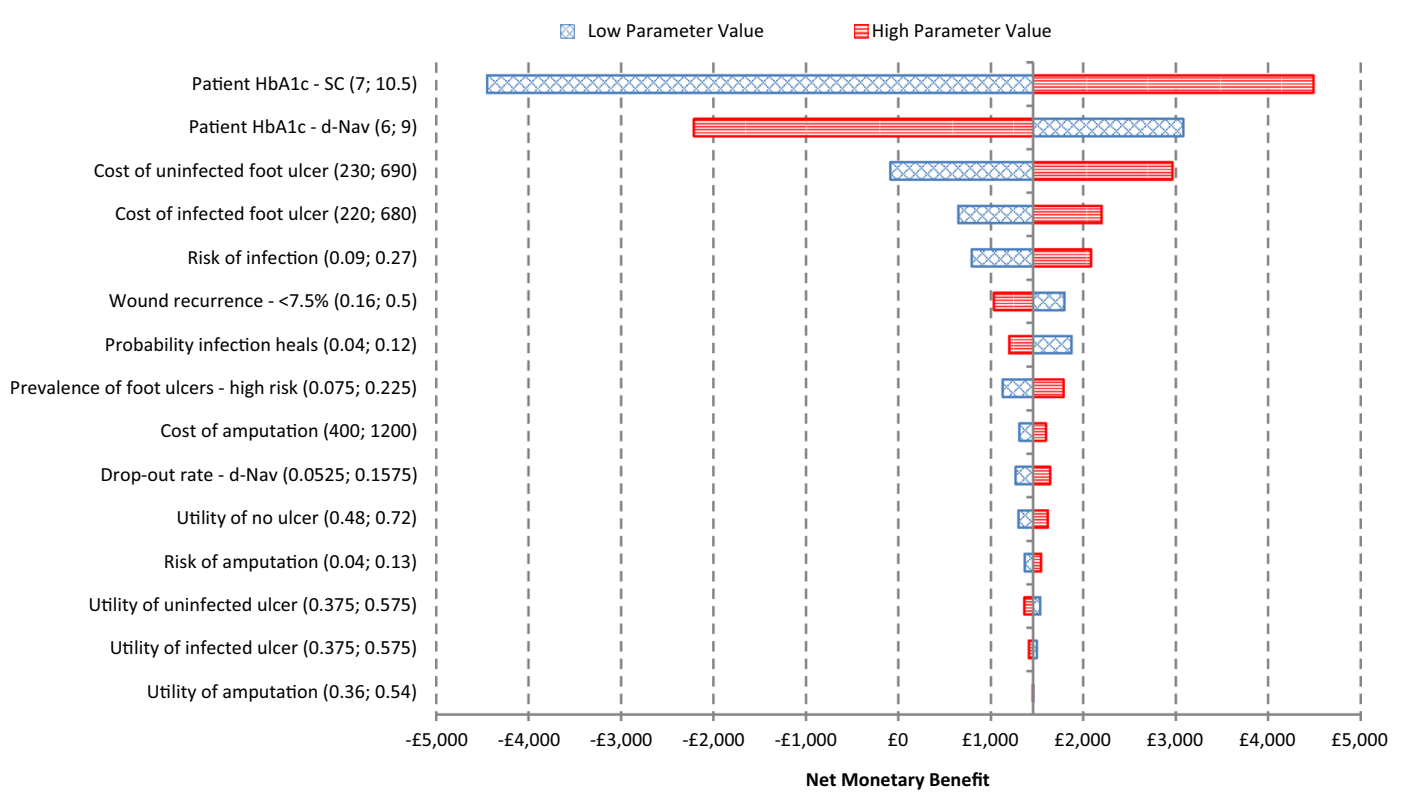

Fig. 6 Tornado diagram of univariate sensitivity analysis. Tornado diagrams can be used to summarize the results of univariate sensitivity analysis. During univariate analysis, the value of each model parameter is individually altered within a predefined range to see the impact on results as measured by the net monetary benefit value. Each parameter is listed on the left, with the extreme values (low and high), that were tested, shown in brackets. The

with guidance issued by the National Institute for Health and Care Excellence (NICE) for its technology appraisal program [27]. To determine the cost-effectiveness of the treatment from a societal perspective, a willingness-to-pay threshold was adopted. Within the model, a cost per QALY threshold of $£ 20,000$ was applied, the lower value of the range formally adopted by NICE $y$-axis represents the base-case analysis. The impact of changes in individual parameter values on the net monetary benefit is shown by the individual bars. If a bar crosses the $£ 0$ threshold, it indicates that $\mathrm{d}-\mathrm{Nav}$ is no longer cost-effective at the parameter value adopted. The graph indicates that three parameters alter the model outputs such that $\mathrm{d}-\mathrm{Nav}$ is no longer cost-effective. The remaining 12 parameters have no impact

[27]. This threshold was used to generate net monetary benefit values with positive values indicating cost-effectiveness at the chosen threshold.

\section{Compliance with Ethics Guidelines}

The analysis in this article is based on previously conducted studies and does not 
involve any new studies of human or animal subjects performed by any of the authors.

\section{RESULTS}

In the analysis, d-Nav was estimated to be cost saving $(-£ 1278)$ and produced more QALYs (0.009) than standard care with a total monetary net benefit value of $£ 1459$ per patient.

The univariate sensitivity analysis is summarized in Fig. 6 . The parameter with the largest impact on results was the underlying mean $\mathrm{HbA1c}$ of both d-Nav and standard care patients. During univariate analysis, the net monetary benefit became negative, indicating that d-Nav is no longer cost-effective, when the mean $\mathrm{HbA} 1 \mathrm{c}$ value for $\mathrm{d}-\mathrm{Nav}$ patients increased from $\quad 7.5 \% \quad(59 \mathrm{mmol} / \mathrm{mol}) \quad$ to $8.2 \%$ $(66 \mathrm{mmol} / \mathrm{mol})$, indicating that $\mathrm{d}-\mathrm{Nav}$ is cost-effective at all values lower than $8 \%$ $(64 \mathrm{mmol} / \mathrm{mol})$. Similarly, the net monetary value became negative when the mean HbA1c for standard care patients decreased from $9.2 \%$ (77 $\mathrm{mmol} / \mathrm{mol})$ to $8.7 \%(72 \mathrm{mmol} / \mathrm{mol})$. Two other parameters also affected the choice of optimal treatment strategy, as determined by the net monetary benefit value, during univariate sensitivity analysis. When the rate of wound recurrence for patients with HbA1c $>7.5 \%$ decreased from $67.30 \%$ to $52.00 \%$, and also when the weekly cost of an uninfected foot ulcer decreased from $£ 463$ to $£ 230$, the net monetary benefit value became negative.

For the scenario analysis, when the scenario was adopted in which all patients started the model in the uninfected foot ulcer state (15\% of the baseline cohort size), then the net monetary benefit increased substantially to $£ 5169$. This equates to per-patient savings of $£ 4858$ and QALY gains of 0.016. Similarly, when the scenario was adopted in which all standard care patients started the model with a HbA1c level of $\geq 9 \%$, the net monetary benefit value again increased substantially to $£ 5310$. This equates to savings of $£ 4992$ per patient and QALY gains of 0.016 .

\section{DISCUSSION}

This analysis indicates that patients with diabetes who have abnormal HbA1c measurements should be targeted with interventions that may improve glycemic control, such as d-Nav, as they appear to provide value for money. Moreover, as highlighted by the scenario analysis, interventions that specifically target patients with very high HbA1c (i.e., $>9 \%$ ) or those who are already with a foot ulcer, are likely to lead to improved patient outcomes and greater cost savings. Treatments that improve glycemic control are estimated to be cost-effective due to the reduction in the incidence of foot ulcers and amputations. This leads to savings as fewer resources are required to treat the underlying conditions. Furthermore, there is a reduction in the number of prescriptions for anti-diabetic agents and blood glucose monitoring devices and test strips and an improvement in patient's quality of life. Given the scale of the burden caused by diabetic foot ulcers, implementing interventions, such as d-Nav, has the potential to generate substantial benefits to the healthcare system. It is important to note that this analysis focused solely on outcomes relating to foot ulcers. However, by improving glycemic control, patients with diabetes and the wider healthcare system are also likely to benefit from a reduction in the incidence of other diabetic complications (e.g., stroke, 
retinopathy), and the magnitude of these benefits could be significant.

Based on the sensitivity analysis that was undertaken, the model results appear to be relatively robust to changes in underlying parameters. The results are most sensitive to changes in the patient HbA1c, which is unsurprising as the model outcomes are largely driven by the reduction in $\mathrm{HbA1c}$ facilitated by d-Nav. This also indicates that interventions are best targeted at patients with very high (>9\%) HbA1c levels.

The HbA1c values, which are the main drivers of the model, are taken from a single-arm study. Ideally, HbA1c would be based on a large-scale, randomized trial that quantifies the difference in HbA1c between patients using standard care and those enrolled on the d-Nav service. However, in this instance, the lack of a control group should not be considered a major limitation, because it is reasonable to use patient $\mathrm{HbA1c}$ at study baseline as the value used for the control group in the model. This is because the value adopted in the analysis is representative of the wider, high-risk population that should be targeted with interventions to improve glycemic control.

The second limitation with the model is that, within the control group, it is assumed that the mean HbA1c remains at $>9 \%$ throughout the full time horizon. In reality, some patients may show improvement; particularly if their treatment regimen is altered (e.g., they are moved onto continuous blood glucose monitoring). However, in clinical practice, a large number of patients will not alter their lifestyle or treatment and, therefore, are likely to remain with unstable HbA1c levels. It is these patients that d-Nav should be targeted at and the ones that are considered in this analysis.
Although the association between HbA1c and diabetic complications is well established, there appears to be a deficit in the research that has been undertaken to quantify the relationship between glycemic control and neuropathy. Therefore, HbA1c could not be linked to the rate of infection, the healing rate for infected ulcers, or the rate of amputation; following a targeted literature search. In reality, patients with HbA1c within recommended ranges are likely to have lower rates for each of these parameters and, thus, improved outcomes overall. It is, therefore, unfortunate that this link could not be quantified in this analysis, and more clinical evaluations are needed to rectify the issue. Furthermore, a target review of the literature was undertaken, and while comprehensive, it may not have captured all available evidence. Therefore, a formal systematic literature review would strengthen the analysis if undertaken.

\section{CONCLUSIONS}

The analysis undertaken here indicates that interventions targeted at patients with diabetes with poor glycemic control, such as d-Nav, may be cost-effective in the prevention and management of neuropathic foot ulcers.

\section{ACKNOWLEDGMENTS}

Sponsorship for this analysis and the article processing charges for publication were funded by Hygieia Inc. for health economics consultancy. The manuscript was also reviewed by the Hygieia team prior to submission. All named authors meet the International Committee of Medical Journal Editors (ICMJE) criteria for authorship for this manuscript, take responsibility for the integrity 
of the work as a whole, and have given final approval to the version to be published.

Disclosures. William Green and Matthew Taylor report sponsorship from Hygieia Inc., during the conduct of the analysis. There are no other conflicts of interest to disclose.

Compliance with Ethics Guidelines. The analysis in this article is based on previously conducted studies and does not involve any new studies of human or animal subjects performed by any of the authors.

Open Access. This article is distributed under the terms of the Creative Commons Attribution-NonCommercial 4.0 International License (http://creativecommons.org/licenses/ by-nc/4.0/), which permits any noncommercial use, distribution, and reproduction in any medium, provided you give appropriate credit to the original author(s) and the source, provide a link to the Creative Commons license, and indicate if changes were made.

\section{REFERENCES}

1. Malanda UL, Welschen LM, Riphagen II, Dekker JM, Nijpels G, Bot SD. Self-monitoring of blood glucose in patients with type 2 diabetes mellitus who are not using insulin. Cochrance Database Syst Rev. 2012. doi:10.1002/14651858.CD005060.pub3.

2. Fowler MJ. Microvascular and macrovascular complications of diabetes. Clin Diabetes. 2008;26(2):77-82.

3. Singh N, Armstrong DG, Lipsky BA. Preventing foot ulcers in patients with diabetes. JAMA. 2005;293:217-28.

4. Nelson EA, O'Meara S, Craig D, Iglesias C, Golder S, Dalton J, Claxton K, Bell-Syer SEM, Jude E, Dowson C, Gadsby R, O'Hareand P, Powell J. A decision analysis for sampling and treating infected diabetic foot ulcers A series of systematic reviews to inform a decision analysis for sampling and treating infected diabetic foot ulcers. Health Technol Assess. 2006;10(12):1-221.

5. NICE. Diabetic foot problems: inpatient management of diabetic foot problems. NICE guidelines [CG119]. NICE, London.

6. Hex N, Bartlett C, Wright D, Taylor M, Varley D. Estimating the current and future costs of Type 1 and Type 2 diabetes in the UK, including direct health costs and indirect societal and productivity costs. Diabet Med. 2012;29:855-62.

7. Stratton MI, Adler AI, Neil HAW, Matthews DR, Manley SE, Cull CA, Hadden D, Turner RC, Holman RR. Association of glycaemia with macrovascular and microvascular complications of type 2 diabetes (UKPDS 35): prospective observational study. BMJ. 2012;321(7258):405-12.

8. NICE. Type 1 diabetes in adults: diagnosis and management of type 2 diabetes [NG17]. NICE, London.

9. NICE. Type 2 diabetes in adults: management [NG28]. NICE, London.

10. Eliasson B, Eeg-Olofsson K, Cederholm J, Nilsson PM. Antihyperglycaemic treatment of type 2 diabetes: results from a national diabetes register. Diabetes Metab. 2007;33(4):269-76.

11. DeFronzo R, Stonehouse A, Han J, Wintle M. Relationship of baseline HbA1c and efficacy of current glucose-lowering therapies: a meta-analysis of randomized clinical trials. Diabetes Med. 2010;27(3):309-17.

12. Esposito $\mathrm{K}$, Chiodini $\mathrm{P}$, Maiorino $\mathrm{M}$, Capuano $\mathrm{A}$, Cozzolino D, Petrizzo M, Bellastella G, Giugliano D. A nomogram to estimate the $\mathrm{HbA1c}$ response to different DPP-4 inhibitors in type 2 diabetes: a systematic review and meta-analysis of 98 trials with 24163 patients. BMJ Open. 2015. doi:10.1136/ bmjopen-2014-005892.

13. Hirsch IB, Bergenstal RM, Parkin CG, Wright E, Buse JB. A real-world approach to insulin therapy in primary care practice. Clin Diabetes. 2005;2:78-86.

14. Daneman D. Type 1 diabetes. The Lancet. 2006;367(9513):847-58.

15. Simmons JH, Chen V, Miller KM, McGill JB, Bergenstal RM, Goland RS, Harlan DM, Largay JF, Massaro EM, Beck RW. T1D Exchange Clinic Network. Differences in the management of type 1 diabetes among adults under excellent control compared with those under poor control in the T1D Exchange Clinic Registry. Diabetes Care. 2013;36(11):3573-7. 
16. Bergenstal RM, Bashan E, McShane M, Johnson M, Hodish I. Can a tool that automates insulin titration be a key to diabetes management? Diabetes Technol Ther. 2012;14(8):675-82.

17. Bastyr EJ, Zhang S, Mou J, Hackett AP, Raymond SA, Chang AM. Performance of an electronic diary system for intensive insulin management in global diabetes clinical trials. Diabetes Technol Ther. 2015;17(8):571-9.

18. Christman AL, Selvin E, Margolis DJ, Lazarus GS, Garza LA. Hemoglobin A1c predicts healing rate in diabetic wounds. J Invest Dermatol. 2011;131(10):2121-7.

19. Dubský M, Jirkovská A, Bem R, Fejfarová V, Skibová J, Schaper NC, Lipsky BA. Risk factors for recurrence of diabetic foot ulcers: prospective follow-up analysis in the Eurodiale subgroup. Int Wound J. 2013;10(5):555-61.

20. Donnelly R, Carr S, Harper R (in press). Diabetes Insulin Guidance System: a real-world evaluation of a novel assistive technology $\left(\mathrm{d}-\mathrm{Nav}^{\mathrm{TM}}\right)$ to achieve glycaemic control in those with type 2 diabetes requiring insulin therapy. Pract Diabetes 2015;32(7):247-252a.

21. Harper R, Donnelly R, Hodish I. Insulin Therapy Transformation in Northern Ireland. Poster session presented at: American Diabetes Association 76th scientific sessions; 2016 June 10-14; New Orleans, LA. https://ada.apprisor.org/epsAbstractADA.cfm? $\mathrm{id}=1$. Accessed 7 June 2016.

22. Rice JB, Desai U, Cummings AKG, Birnbaum HG, Skornicki M, Parsons NB. Burden of diabetic foot ulcers for medicare and private insurers. Diabetes Care. 2014;37(3):651-8.

23. PSSRU. Unit costs of health and social care. Canterbury: The University of Kent; 20142014.
24. National Institute for Health Research. Case study: Self-monitoring of blood glucose in type 2 diabetes. http://www.nets.nihr.ac.uk/. Accessed 10 Sept 2014.

25. Regional Drug and Therapeutics Centre. Cost Comparison Charts-July 2014. http://gmmmg. nhs.uk/docs/cost_comparison_charts.pdf. Accessed 15 Sept 2014.

26. Flack S, Apelqvist J, Keith M, Trueman P, Williams D. An economic evaluation of VAC therapy compared with wound dressings in the treatment of diabetic foot ulcers. J Wound Care. 2008;17(2):71-8.

27. NICE. Guide to the methods of technology appraisal 2013 [PMG9]. NICE, London.

28. Marston WA, Hanft J, Norwood P, Pollak R. The efficacy and safety of Dermagraft in improving the healing of chronic diabetic foot ulcers: results of a prospective randomized trial. Diabetes Care. 2003;26(6):1701-5.

29. Morbach S, Furchert H, Gröblinghoff U, Hoffmeier $\mathrm{H}$, Kersten K, Klauke GT, Klemp U, Roden T, Icks A, Haastert B, Rümenapf G, Abbas ZG, Bharara M, Armstrong DG. Long-term prognosis of diabetic foot patients and their limbs: amputation and death over the course of a decade. Diabetes Care. 2012;35(10):2021-7.

30. British National Formulary. https://www.BNF.org. Accessed 5 Sept 2014.

31. HSCIC. Prescribing for Diabetes, England-2005-06 to 2012-13. http://www.hscic.gov.uk/catalogue/ PUB11422. Accessed 15 Sept 2014. 